Pfhrp-2 gene deletion is implicated in limiting RDT sensitivity. Studies evaluating Pfhrp-2 and Pfhrp-3 deletion and the amino acid sequence diversity has not been investigated in Nigeria. We therefore hypothesised that malaria parasites in Nigeria are lacking Pfhrp-2/Pfhrp-3 genes with variable amino acid repeats sequences.

Methods The study was part of a prospective cohort study evaluating RDTs performance. We pooled 66 samples comprising false negatives $(n=31)$ and true positives $(n=35)$ to elucidate Pfhrp-2/Pfhrp-3 gene deletion, RDT cross-reactivity with Pfhrp-3 antigen and amino acid sequence diversity. The 18SrRNA, msp 1, msp2 and glurp genes were amplified to establish active Plasmodium falciparum infection and the exon-2 regions of Pfhrp-2 and Pfhrp-3 genes were amplified to determine the presence or absence of Pfhrp-2 and Pfhrp-3 genes. Isolates with conserved Pfhrp-2/Pfhrp-3 were sequenced.

Results All 66 samples were positive for 18SrRNA, msp1, msp2 and glurp, indicating active $P$. falciparum infection. However, $16.7 \%$ and $6.0 \%$ of the samples were lacking Pfhrp-2 and Pfhrp-3 genes. Of the false negative samples, $25.8 \%$ and $12.9 \%$ has Pfhrp-2 and Pfhrp-3 deletions. Three Pfhrp-3 conserved antigens cross reacted to give RDT positive results. An extensive diversity in the amino acid sequence was observed.

Conclusion Plasmodium falciparum parasites in Nigeria lack Pfhrp-2 and Pfhrp-3 genes. However, the proportion of deletions is low compared to reports from other malaria-endemic regions. In addition, a high amino acid tandem repeat was observed. A combination of pLDH and Pfhrp-2 based RDTs is recommended for accurate malaria diagnosis.

\section{PO 8608 ESTABLISHED PARTNERSHIPS OF THE UNIVERSITY OF CAPE VERDE WITH THE UNIVERSITY OF LEICESTER, UK AND THE INSTITUTE OF HYGIENE AND TROPICAL MEDICINE, PORTUGAL}

${ }^{1}$ Isabel | Araúijo*, ${ }^{2}$ Sandra Beleza, ${ }^{3}$ Maria Do Rosário Martins. ${ }^{1}$ Faculdade de Ciencas e Tecnologia, Universidade de Cabo Verde, Cape Verde; ${ }^{2}$ Department of Genetics and Genome Biology, University of Leicester, UK; ${ }^{3}$ nnstituto de Higiene e Medicina Tropical, Universidade Nova de Lisboa, Portugal

\subsection{6/bmjgh-2019-EDC.163}

Background The University of Cape Verde (Uni-CV) was founded in 2006 and since then has developed an effective international strategy that is enhancing the teaching and research culture of the university. To build capacity in the public health field, Uni-CV has established collaborations with the University of Leicester (UoL), UK, and with the Institute of Hygiene and Tropical Medicine (IHTM - NOVA University), Portugal. These follow different approaches.

Methods Different type of capacity building initiatives focused on researchers and postgraduate students.

Results The collaboration with UoL was established in 2016 and builds on funded research programmes in infectious disease and evolution of antimicrobial drug resistance (AMR). These programmes have allowed technology development and transfer, which offer the opportunity for undergraduate students to lead laboratory-based research projects at Uni-CV. In these two years, we have successfully characterised the epidemiology and patterns of AMR underlying Helicobacter pylori infections in Cape Verde. Beyond the research outputs, we have trained four undergraduates in laboratory-based microbiology at Uni-CV. From this experience, we are currently designing new sustainable higher education programmes aligned with the MSc in Public health at Uni-CV that aim to support Uni-CV lecturers in the supervision of postgraduate students, either independently or in collaboration with UoL researchers.

The collaboration with IHTM was established in 2008 with the main aim of developing the research capacity of Cape Verde researchers and health professionals in infection and vector-born diseases and in bioethics. These activities have also led to collaborative research in these areas. Since then, six training courses were taught that benefited $\sim 20$ Uni-CV lecturers and $\sim 100$ government professionals.

Conclusion Although these collaborative approaches are different in nature, they have been successful in the training of health professionals, researchers and technicians in Cape Verde and have contributed to the establishment of mutually beneficial research programmes.

\section{PO 8609 PREVALENCE AND RISK FACTORS FOR GLUCOSE-6- PHOSPHATE DEHYDROGENASE (G6PD) DEFICIENCY IN TWO $P$. VIVAX MALARIA-ENDEMIC AREAS IN SUDAN}

Muzamil Mahdi Abdel Hamid ${ }^{1 *}$, Musab Albsheer', Mohamed Muneer', Lina Altinae', Andrew A Lover'. 'Institute of Endemic Diseases, University of Khartoum, Sudan; ${ }^{2}$ Institute for Global Health Sciences, University of California, San Francisco, United States of America

\subsection{6/bmjgh-2019-EDC.164}

Background Plasmodium vivax malaria is a major health problem in Sudan and the parasite has become widely distributed in the recent years. The WHO recommends the use of primaquine as radical cure for liver dormant stage, the hypnozoite. However, prior its use, a test for Glucose-6-phosphate Dehydrogenase (G6PD) should be performed. The objective of the current study was to determine prevalence and risk factors for G6PD deficiency in two $P$. vivax malaria-endemic areas in Sudan.

Methods A cross-sectional study recruiting 557 subjects from two malaria-endemic areas in Sudan was conducted. Demographic data and blood samples were collected. G6PD activity was measured by spectrometry using SPINREACT enzymaticUV kit.

Results The measured G6PD activities for both sites ranged from 0.6 to $37.7 \mathrm{U} / \mathrm{g} \mathrm{Hb}$, with a median value of $12.8 \mathrm{U} / \mathrm{g}$ $\mathrm{Hb}$. There was a significant difference in enzyme activity by study site $(\mathrm{p}<0.001)$, but not by sex $(\mathrm{p}=0.91)$. Overall, across the two study sites, $22(3.9 \%)$ is G6PDd $(<30 \%)$. Prevalence of G6PDd $(<30 \%)$ in Khartoum is $1.8 \%(4 / 230)$ compared to $4.8 \%(16 / 327)$ in New Hafla. In univariate analysis predictors of G6PDd were study site (odds ratio of G6PD activity $<3.8$, Khartoum relative to New Halfa $=0.22$ (95\% CI: 0.08 to $0.66), p=0.006)$, and recent antibiotic use $(\mathrm{OR}=2.45 \quad(95 \%$ CI: 1.1 to 5.5$), p=0.027)$. In multivariate analysis, the only factor that was significant was the individual's weight in kilograms, with an OR of 0.97 (95\% CI 0.95 to $0.99, \mathrm{p}=0.014$ ). Conclusion G6PD deficiency is less prevalent among Sudanese population and this indicates that the use of primaquine for radical cure of $P$. vivax malaria is safe. 